\title{
Children's experiences of living with a parent with mental illness: A systematic review of qualitative studies using thematic analysis.
}

\begin{abstract}
Introduction: This paper brings together what is known about what it is like for children who live with a parent with a mental illness with a view to strengthening our understanding of their experiences. This paper presents an update on previous reviews that were completed in this area and used a systematic approach and stringent inclusion/excluiosn criteria to ensure that the voices of children were central in the included papers. A systematic review of this nature could not be located in the literature.
\end{abstract}

Aims: This paper presents the findings of a systematic review which explored the experiences of children who were affected by parental mental illness.

Methods: CINAHL, PubMed, PsychINFO, Pubmesh, and Embase were searched for qualitative studies which explored children's experiences and eight studies were included following review and quality appraisal. Pertinent data were extracted, coded and analysed using a thematic approach.

Results: Four themes emerged from the analytic process which described the children's understanding of mental illnesss, their relationship with their parents, their coping strategies and their social connections.

Implications for Practice: Mental health nurses need to recognise the psychosocial needs of children and to acknowledge the wider impact mental illness has of the family.

\section{Accessible Summary}

\section{What is known about the subject?}

- There are many qualitative studies that explore what it is like for children who live with a parent who has a mental illness.

- These studies are sometimes criticised because they have small sample sizes which limits their application.

\section{What this paper adds to existing knowledge?}

- We conducted a systematic review of qualitative papers with an aim to strengthening our understanding of what it is like for children who live with a parent who has a mental illness.

- We used stringent criteria to make sure that only the voices of children affected by parental mental illness were included in the review.

- In addition, the paper presents a timely update on previous reviews completed in this area. 


\section{What are the implications for practice?}

- The findings of this review highlight the impact that parental mental illness has on children and the important role that mental health nurses can play in maximising opportunities for building resilience in affected children.

- Mental health nurses are in a key position to provide timely and age appropriate information and support to both parents and children to assist in the development of appropriate coping and support mechanisms.

\section{Relevance Statement}

This systematic review brings together what is known about the experience of children who are affected by mental distress. There is evidence to suggest that mental health nurses have a lack of interest, commitment, knowledge and experience of the children of people who use the mental health services. In order to provide for children's' specific needs, a clear understanding of their needs is imperative.

\section{Key Words}

Child, adolescent, parental mental illness, systematic review, qualitative research, thematic analysis.

\section{Citation:}

Yamamoto, R. \& Keogh, B. (2017), Children's experiences of living with a parent with mental illness: A systematic review of qualitative studies using thematic analysis. Journal of Psychiatric \& Mental Health Nursing. Accepted Author Manuscript. doi:10.1111/jpm.12415 


\section{Introduction}

Many people who experience mental illness over the course of their lives may also be parents. In Australia, for example, $20.4 \%$ of mental health service users have dependent children (Maybery et al. 2009, O'Brien et al. 2011). While in Canada, it has been calculated that just over $12 \%$ of children under the age of 12 live with a parent who has a mental illness (Gladstone et al. 2014). In the United Kingdom, approximately 2.5 million children live in families affected by parental mental illness (Tunnard 2004). Overall, over $67 \%$ of women and over $75 \%$ of men with serious mental illness are parents (Nicholson et al. 2004). The literature suggests that children who have a parent with a mental illness, might have greater cognitive, emotional, and behavioural difficulties, potentially leading to diagnosable psychiatric problems in later life (Göpfert et al. 1996, Beardslee et al. 1998, Gladstone et al. 2014). Moreover, when children affected by parental mental illness have been identified as 'at risk', this status pursues them into adulthood and the coping strategies they use to manage their lives may have negative social consequences (Andres-Lemay et al. 2005, Mordoch \& Hall 2008, Gladstone et al. 2011). Furthermore, children affected by parental mental illness are more likely to live with a single parent or are at a greater risk of being taken into foster care (Leschied et al. 2005). These studies evidence that parental mental illness could affect children's' health, social lives and family structures. Despite this, children in these situations are often overlooked in mental healthcare settings and have been described as being 'hidden' and 'invisible' (Fudge \& Mason 2004, Maybery \& Reupert 2009). This is because many mental health professionals do not consider or are blind to the needs of service users who are in their care who also have children (Hetherington \& Baistow 2001, Gladstone et al. 2006). Furthermore, a paucity of studies which explores the perceptions and experiences of these children may perpetuate children's exclusion from mental health care practices (Gladstone et al. 2006, Östman 2008, Mordoch \& Hall 2008, Kaimal \& Beardslee 2010, Trondsen 2012).

Qualitative studies have offered an insight into children's experiences of parental mental illness; however they often have small sample sizes limiting their application and generalisability. Qualitative systematic review offer a way of bringing together research on a topic from primary qualitative studies (Seers 2015) which can strengthen the methodological rigor of individual studies. A literature review conducted by Gladstone et al. (2011) provided a review of qualitative studies from 1997 to January 2010. However this was not a systematic review and declared a number of methodological limitations. This paper uses a systematic review to provide a more up to date and more methodologically robust review of children's experiences of parental mental illness. A comprehensive knowledge of the experiences and perceptions of children whose parents 
have a mental illness is necessary to inform mental health nursing practice and research. Qualitative synthesis has been described as being either aggregated or interpretive (Noblit \& Hare 1988). Our approach sought to aggregate data from similar studies and provide a summary of their findings using thematic analysis (Seers 2012). This can be understood as being under the umbrella of qualitative descriptive research and as such a level of interpretation has been achieved.

\section{Method}

\section{Selection Criteria}

Peer reviewed qualitative studies which used phenomenological, grounded theory, descriptive or ethnographic research design and which explored the experiences of children who lived with one or more parents with a mental illness were selected for inclusion in this review. Quantitative studies, mixed method studies, literature reviews, questionnaires and surveys and non-English papers were excluded. Children were defined as those research participants who were eighteen or under at the time of participating in the research.

\section{Search Strategy and Selection Method}

The electronic databases CINAHL, PubMed, PsychoINFO, Embase and MEDLINE were searched in February 2015. A systematic search strategy was created using the PEO template developed by Bettany-Saltikov (2012). The search string was ramified of five categories, which were (Participants) 'children', (Exposure) 'parents', 'mental health problems', (Outcome) 'perceptions', and (Type) 'qualitative method'. The search strategy used is available on request. Following the rule of transparency and replication of the process of selecting eligible papers, three sequential steps were carried out in this stage. Firstly, all the papers retrieved from the databases were screened by title and abstract. Secondly, the remaining papers from the initial screening were read full-text in order to adhere to consistency in the inclusion criteria. In the case of ambiguous papers a consensus was reached by both reviewers in terms of their suitability for inclusion in the review.

\section{Quality assessment, Data Extraction and Thematic Analysis}

The quality assessment process applied the framework of the EPPI Centre Tool developed by Brunton et al. (2011). The score provides $0,0.5$, and 1 depending on clarity of the answers to the questions posed, with a maximum possible score of 10 . The reviewers decided to include either high or medium quality papers to enhance credibility. The review was conducted by two people individually and then any disagreements were discussed where applicable. Data was extracted by the primary author and then checked 
by the second author. This involved extracting data pertinent to the review aims and objectives using a pre-designed template. Once extracted, a process of coding was completed and similar codes were grouped together to form themes, which were then further reviewed and collapsed until four main themes emerged.

\section{Results}

An overview of the review process can be seen in figure 1. In total, 6988 citations were retrieved from electronic sources, 15 Studies were excluded because they were duplicates. Following title and abstract review, 6954 citations were excluded as they did not meet the review criteria for inclusion and 19 full text studies were reviewed. Eight studies were excluded at this point because they were not relevant $(n=2)$, not qualitative design $(n=3)$ or they did not meet the inclusion criteria $(n=3)$. A further two papers were also excluded after this process because of unclear outcomes and design issues respectively. Nine studies were brought forward for quality review with one paper being excluded after receiving a low quality score. In total, 8 papers were taken forward to the data extraction stage. An overview of the selected papers is presented in table 1.

Following the analytic processes, four themes emerged from the extracted data:

(1) Children's understanding of parental mental illnesss

(2) Children's relationship with parents

(3) Coping strategies

(4) Social connections of children

[Insert figure 1 here]

[Insert table 1 here]

\section{Children's understanding of parental mental illnesss}

The first theme outlines the children's understanding of their parent's mental illness. The level of understanding was different for children depending on factors such as developmental stage, a family policy which told children about their parental mental illness or not, and a sense of desire to seek information about their parents mental health. Most young children had not been told about their parents' mental health and therefore, they tended to describe the condition of their parents visually.

An 8-year-old boy talked about his mother, 'She just cries. And then she just sits there. That is depression.' A 12 year-old girl described her father's symptoms of schizophrenia, 'He just ...talks about nonsense things like aliens and wicked stuff and all that weird kind of jazz that, you know, just don't really jive' (Riebschleger 2004, p.28).

According to Riebschleger (2004), only a few children in the study were told about their parents mental illness, whereas the others rarely received appropriate explanations. Hence, the children gradually recognised parental differences in speech and behaviour by comparing them to other parents. This vague understanding motivated children to seek further information about what was happening to their parents although this was difficult. 
There were limited opportunities for the children to access this information and for the most part parents or other adults did not answer the children's questions. This confusing situation contributed to an increased sense of frustration and isolation for the children. A few children were able to access information by reading literature and participating in a volunteer group. Limited information about parental mental illness made children confused as how to deal with their parents' behaviour and was a source of anxiety about themselves and their future. The provision of information to children about parental mental health was important and contributed to a better understanding of their parent's condition. It also lessened or eliminated the children's anxiety and negative emotions.

Children who were knowledgeable about the illness were better also to interpret their parents' behaviours as indicators of their illnesses. Their knowledge helped the children to experience less uncertainty about what was happening and made their painful emotions less intense (Mordoch \& Hall 2008, p. 1131).

Although struggling with getting information about and understanding parental mental illness, most children realised that mental illness was not as easy to treat and 'cure' as physical health problems. Some children were unsure of whether their parent took medication but most had negative feelings towards parents taking medicine. In terms of treatment, children expressed feelings of dissatisfaction with medical staff because the children didn't feel respected and there appeared to be inadequate communication between the medical staff and their parents. In addition, children's lack of information about parental treatment made them feel excluded from the parental care plan.

'I go to the doctor with my mum. She was supposed to go today but she didn't get up. But then I hope like when we go there they don't just give her antidepressants and tell her to go home, you know what I mean, because it's not the answer and most doctors now just give you them an decent life. But it's not on really. What they should do is just help her a bit more and speak to her. She's not well, but she's not an invalid (Abbie)' (Grant et al. 2008, p. 276).

Parental hospitalisation was one of the big concerns for children and it left them in the dark about their future. The children's interpretation of hospitalisation differed among individuals. The majority of perceptions of hospitalisation were linked to the exacerbation of parental mental state whereas, in the studies of Riebschleger (2004), and Mordoch and Hall (2008), some children linked it to parents' death. Moreover, parental hospitalisation led to a possibility of children being separated from parents. There were two types of separation: being put into foster care, and being taken care of by relatives. The former was an unpopular choice for children, because they felt uncomfortable with unfamiliar people and environments when in foster care, while the latter was a better choice for some children because the children had been warmly cared for by relatives. On the other hand, some children had positive perceptions of parental hospitalisation because it brought them a sense of ease, safety and relief from parental distress. Moreover, it was unnecessary to worry about their parents because they were in a secure environment. 
Several children associated the hospital as a place where people died, e.g., 'Grandpa died in the hospital,' and "sometimes it (a psychiatric disability) may kill you. What I know about mental illness is when you get real sick, you have to go to the hospital and then you die' (Riebschleger 2004, p.29).

Worry about the parent was often related to an existential fear: 'what is going to happen to me when my parent does not make it?' (Van Parys \& Rober 2013, p. 334).

The children expressed a range of emotions about living with a parent with mental illness. These were both positive and negative and included feelings such as fear, confusion, exhaustion, distress, powerlessness, love, hope, and a sense of responsibility. Children tried to understand parental mental illness as being the same as any other illness and nothing to feel embarrassed about. However, they realised some people such as neighbours and even relatives stigmatised them and their parents. Therefore, parental illness sometimes caused anger in their children.

They have a family member, in this case a parent, who behaves differently. Sometimes this makes the child angry at the parent's peculiar behaviours, and even with the parent him/herself. Children of all ages often explain in the narratives that 'People are afraid of persons with a mental illness (Östman 2008, p. 357).

\section{Children's relationship with parents}

Parental mental illness sometimes caused family disruption such as parental arguments and divorce which had a huge impact on children. Family disruption sometimes caused a weak connection between children and their parents and relatives. Some children wished that they lived with a healthy parent instead of a parent with mental illness because of the more comfortable environment provided including psychological, physical and financial aspects. The capacity to parent was also damaged by parental mental illness which deprived parents of their confidence and authority in being parents. Likewise, children worried about their future because they feared losing a parental model. It often caused parental role reversal such as doing housework and taking care of other siblings, which put more burden on children's shoulders, especially for older children.

'We don't have much contact with them anymore. My auntie used come but she doesn't now. I don't see her now. If my mum's not well, well, it's...difficult. Dad tried when he was there but he'd get you know, upset and angry...sometimes he'd go to his room' (Child stakeholder, 17 yrs, Mother with psychosis) (Bee et al. 2013, p.6). 
'It's kind of difficult/different to distinguish who's the parent and who's the child sometimes. Um (Pause), just because my mother has a lot of needs it's difficult for her to be (Pause) the caregiver, and I tend to be more of the caregiver. She can't be there emotionally for me' (Meadus \& Johnson 2000, p.386).

On the other hand, children wanted to spend more time with their parents. Riebschleger (2004) suggests that children think a "good day" is when their parents can interact with them more often. Younger children, especially, tended to engage with their parents more than older children. Despite having difficulties at some points in their lives children were able to understand their parent's hardship and accept their difficult living situation based on their sense of love and compassion for their parents. Based on these emotions, children often tried to hide their negative emotions and tried not to be a burden on their parents which they believed made their parents' condition stable. Parental mental illness also contributed to a sense of responsibility and blame in children. Some children felt that they were helpless, and that their behaviour worsened their parent's mental health. Other striking emotions were loneliness and isolation. Children felt that they had no one to talk to because they either feared stigmatization or were told by their parents not to speak about the parent's mental health.

\section{Coping strategies}

The children used a variety of coping strategies in order to deal with their negative emotions and to accept their parent's mental illness. The most common coping strategies were doing some activities such as playing a game or sports, and talking to somebody to let their stress out as well as expressing their feelings and experiences. However, the types of coping strategies used depended on the children's ages which is demonstrated in the following two quotations.

Because younger children had limited options, they were more likely to go to their rooms, sleep, play, choose to think of happy times, read, and take comfort in stuffed animals and pets (Mordoch \& Hall 2008, p.1133).

Older children could go get away, to 'hang out and chill' with friends, go to sleepovers, go to the mall or work or drive a car in an effort to develop their own lives (Mordoch \& Hall 2008, p. 1133).

Mordoch and Hall (2008) described two types of coping strategy using the terms "finding the rhythm" and "maintain the frame", which meant respectively coping with parental issues actively and taking a break from parents. This involved either offering the parents comfort and affectionate behaviour, improving the parent's environment by doing household chores or cooking a meal or ignoring, avoidance and silence when the children were taking a break. Some children took a farsighted view that their life was different from their parents' life. Taking such a practical approach made children more comfortable in creating a distance from their parents. Another coping strategy involved 
children using alcohol and drugs. It was assumed that some children found it too difficult to manage issues of parental mental illness, including their own emotions, and they found a way to escape from their harsh situation through illegal means. Some children made comparisons between themselves and their peers and identified the positive aspects of living with a parent who had a mental illness. They felt that their experiences had contributed to their maturation and growth.

\section{Children's social connections}

The fourth theme focuses on the social connections of children where children encountered social interaction problems as a result of parental mental illness. Regarding the challenge of living with a parent with a mental illness, most children looked for help outside their home and were likely to rely on people whom they trust. The children thought seriously about the importance of having trustworthy friends or relatives in order to just be with them or be listened to about their concerns in their lives. These people made children relaxed and eased their distress.

Participants spoke of the value of friends as a source of support, whether or not they chose to talk with them about their parent's illness (Garley et al. 1997, p.101).

Some children preferred to talk about their parental mental illness to friends who had similar experiences to them. It made the children more comfortable and they felt empathy for each other. Not all children preferred to share their experiences with their friends because they had experienced other children gossiping about their parents or they were afraid of being stigmatized in other ways. Parental mental illness was often described as a family secret; younger children, especially, were influenced by parents' policy of not talking about family problems outside the home. Even within a family, in some cases, it seemed to be taboo to talk about parental mental illness. With regard to this, children respected other family members' feelings, and cared for each other in nonverbal ways. When children and their parents made a decision to share their experience, the selection of a suitable person was carefully made, and then the selected people were usually friends, counsellors and teachers.

To preserve themselves, the children sometimes selectively shared their experiences with trusted others, including their parents, peers, friends, and helpers such as counsellors and teachers (Mordoch \& Hall 2008, p.1134).

Children selectively shared their worries with some friends, or a specific teacher, but not with others (Van Parys \& Rober 2013, p. 335).

However, although friends, teachers and counsellors were regarded as suitable people to share experiences of parental mental illness, there were a variety of preferences and opinions among children. For example friends of the same gender and certain teachers 
were favoured among the children. A lack of supports and services from mental health professionals was expressed by children. In addition, there were reports of isolation from school and other social networks due to stigma or because they had insufficient time due to parental caring activities.

\section{Discussion}

The aim of this review was to synthesise the findings of qualitative studies that explored the experiences of children whose parents had a mental illness. The findings of the review have been summarised into four main themes. It is clear from the analysis that the children and adolescents in these studies experienced a certain amount of distress when one or both parents were treated for mental illness. This distress often emerged in response to a lack of knowledge about their parent's condition and many of them experienced loneliness and isolation, which impacted on their academic achievements and their wider social networks such as making friends. While our review only included 8 studies, the themes that emerged correspond with those of Gladstone et al. (2011) who completed a literature review on the same topic with 20 studies. The current review however builds on Gladstone et al.'s (2011) paper with the inclusion of two additional studies. In addition, the current review sought to focus on the voice of the children affected by parental mental illness, a limitation acknowledged by Gladstone et al. (2011). This was achieved through the systematic processes applied and defined inclusion and exclusion criteria where studies were excluded when there was ambiguity in terms of the data presented. Furthermore, quality appraisal of the studies ensured that low quality papers were also excluded which enhanced the rigor of this review. The exclusion criteria used for this review also meant that studies with participants over the age of 18 were not included which may explain the differences in the number of included studies when compared to Gladstone et al. (2011). The ages of the children in this study ranged from 5 - 18 and while this presents limitations in terms of analysis, it does provide a description of the children's experience trajectory and how we might expect them to react over time. However, the heterogeneity of this sample is acknowledged and further analysis of the extracted data taking a stratified approach is warranted although was beyond the scope of this review.

There are a number of points about the included studies that are worthy of discussion. The sample sizes used in the studies included ranged from 3 participants to 22 participants (mean sample size 11.3). This review has attempted to address the limitations inherent with smaller sample sizes by bringing together research from primary studies and combining and analysing their findings thus enhancing and strengthening their application and rigor. This increases the number of participants to 91 representing 41 males and 50 
females. In terms of methodology a range of qualitative approaches were used across the studies, however in some of the papers there is a lack of detail about the philosophical underpinnings of the methodology and the nature of the relationship between the researchers and the data. This may limit the transparency of the studies and the ability to replicate them under similar circumstances. Furthermore, there is also a lack of detail about the data analytic processes in some of the papers and adherence to the COREQ (Tong et al. 2007) or similar guidelines for reporting qualitative research may enhance future studies in the area. While this review has reported on the experiences of children affected by parental mental illness, there is a diversity of methodological approaches which may detract from the review findings. It is worthy of note that all but one of the studies took steps to enhance the rigor of their findings. In terms of parental mental illness, a range of mental illness diagnoses were reported; mood disorder (Garley et al. 1998); bipolar disorder and unipolar disorder (Meadus \& Johnson 2000); bipolar affective disorder, major depression, schizophrenia and post-traumatic stress disorder (Riebschleger 2004); mental health problems (Grant et al. 2008); Schizophrenia and affective disorder (Ostman 2008); bipolar disorder, depressive disorder, schizophrenia, borderline personality disorder (Bee et al. 201 2013); schizophrenia, depression and bipolar disorder (Mordoch and Hall 2008); depression (Van Parys \& Rober 2013). While it is not our intention to interpret mental health problems as purely medical, future studies might benefit from exploring the effect of parental mental illness on children using more homogenous samples.

This review underscores the importance of family focused practices as described by Foster (2012) among mental health nurses. In particular, the provision of age appropriate information to children and adolescents about their parent's mental illness. Trondsen (2012) argues that in the absence of information, parental illness may be extremely frightening and painful for children. In addition, when parents are admitted to hospital, these events may appear catastrophic to some children. The majority of children had not been explicitly told about their parents' mental illess, and most of them recognised it gradually by comparing their parents to other parents. This limited information about parental mental illness caused children a sense of concern, fear, and frustration. In addition, knowing only a part of the information makes it difficult for children to predict their parents' behaviour (Pretis \& Dimova 2008). As also evidenced by the findings, it misled children into worse imaginings about their parents' health, such as death. From the parents' point of view, it has been argued that some parents want their children to understand their mental illness so that the children do not feel that they are responsible for the parents' problems or the treatment that they receive (Stallard et al. 2004). However, 
the provision of age-appropriate information for children, which explains parental behaviour and emotions, is regarded as challenging. From the findings, there was a gap between younger children and older children in relation to the level and accuracy of understanding of parental mental illness. Therefore, it is clear that how much and how deep the information provided depends on children's capacity to understand and their stage of development. On the other hand, Stallard et al. (2004) argue that the provision of information about parental mental illness might not be always right for all children. Interestingly, the eligible papers for this review emphasised only the necessity of provision of information about parental mental illness as children wish. In comparison, Stallard et al.'s (2004) study provided evidence that a few children are afraid of getting information about their parents' mental illness because such information might become an unnecessary burden and some parents hesitate to provide it for that reason. A psychoeducation programme on mental health, which responds to the children's age, within a hospital or organisation where their parents belong might help them to understand parental mental illness. In addition, it might provide opportunities for children to find peer support from other children in similar situations. This approach could also be useful to help children understand parental hospitalisation, which had a huge impact on the children's lives. According to Pretis and Dimova (2008), repeated parental hospitalisation and separation from their parents for unidentified terms of time can lead to children's separation anxiety and extreme concern about their parents. These negative emotions may be alleviated if children are helped to understand the purpose of hospitalisation. Therefore, the role of mental health nurses might be to support and help parents to talk about their mental illness to their children and help the parents to be better equipped to support their children during difficult times. Furthermore, mental health nurses are in an excellent position to help children explore their concerns about their parents while encouraging parents to maintain contact during periods of hospitalisation which may minimise any sense of fear and exclusion that the children experience.

Most children in this review emphasised the importance of having people that they could trust, whether they talked about their parental mental illness or not. Sharing experiences of parental mental illness depended on the intention of children or parents. Some children preferred to talk about such experiences to their friends in order to be freed from distress. However, they also found that this was risky because they had no idea of how others would respond, or if they would help, and did not want to be perceived as different (Gladstone et al. 2011). In fact, some children had experienced gossip about their parents. On the other hand, some children felt more comfortable sharing their experiences with children who had a common experience because they could easily understand each other with empathy. This is because peer support might be a less judgmental environment 
to talk about sensitive issues (Hayman 2009). Fjone et al. (2009) also argue that children look for people who are in similar situations because this helps them to feel 'normal'. It can be assumed that children think that mental illness is associated with stigma; therefore, secrecy and shame often prevent them from sharing their parental mental illness experiences with others (Aldridge \& Becker 1993). Sharing parental mental illness is a sensitive and private issue; therefore, they are often explained as a family secret or shared only with selected people. From the findings, some parents were unwilling for their children to talk about their mental illness and this attitude was mostly respected by their children. While selective sharing was generally carried out among children's friends, teachers and healthcare professionals, this also depends on children and parents' preference. For example, according to Mordoch and Hall (2008), although counsellors were seen as helpful, some children did not think counselling was helpful, and the children wished to be treated the same as their peers. Van Parys and Rober (2013) explain that even among family members, it is difficult to talk about problems. This is supported by Rober (2002) who suggests that there is sometimes a reluctance to talk about a family member experiencing distress for fear of hurting their family member even more. Studies demonstrate that sharing parental mental illness in a peer group increased children's resilience and decreased their risk of mental illness (Orel et al. 2003, Pitman \& Matthey 2004). Therefore, allowing children to voice their opinions in terms of the most suitable support mechanism in partnership with parents may lead to more successful outcomes.

\section{Limitations}

It may have been beneficial to include quantitative or mixed methods studies in this review. However, as the review was completed as part of an MSc in Mental Health, the timeframe allowed for completion did not permit this. Likewise, the inclusion of grey and other non-peer reviewed sources of data may also enhance the review but they were not included for the same reason. Future reviews may be able to develop the themes identified in this review by sourcing grey and other non-peer reviewed work.

\section{Conclusion and recommendations}

The findings of this review highlight the impact that parental mental illness has on children and the important role that mental health nurses can play in maximising opportunities for building resilience in affected children. Key issues of concern include the provision of timely and age appropriate information and the provision of support to both parents and children to assist in the development of appropriate coping and support mechanisms. While parents and their children must remain central, other key stakeholders needs to be involved as required to help sustain a supportive network as appropriate while listening to the voices of both parents and children. Lauritzen and Reedts (2013) argue that mental health professionals in adult psychiatry have a lack of interest, commitment, knowledge 
and experience of the children of people who use their services. This lack of motivation reflects in children's concern, mistrust and disappointment towards them. Therefore, there needs to be a more concerted effort in the provision of family focused approaches which does not rely on individuals but is embedded across and within mental health services through careful planning. There is also a need to raise awareness among mental health nurses of children's needs through training and educational programmes which promote children's mental health and sensitivity towards children's issues. Additionally, there should not only be reliance on mental health nurses' skills and individual motivation, but also mental health services need to make an organisation-wide attempt to promote this effort. While there is a desire to promote person centred and holistic practices within the mental health services, these approaches need to radiate to those individuals that are central to the service user's recovery. 


\section{References}

Ahlström B.H., Skärsäter I. \& Danielson E. (2011) Children's View of a Major Depression Affecting a Parent in the Family. Issues in Mental Health Nursing 32, 9, 560-567.

Aldridge J. \& Becker S. (1993) Children who care: Inside the world of young carers. Loughborough University.

Andres-Lemay V. J., Jamieson E. \& MacMillan H. L. (2005) Child abuse, psychiatric disorder, and running away in a community sample of women. Canadian Journal of Psychiatry 50, 11, 684.

Beardslee W. R., Versage E. M. \& Gladstone T. R. G. (1998) Children of affectively ill parents: A review of the past 10 years, Journal of the American Academy of Child \& Adolescent Psychiatry 37, 11, 1134-1141.

Bee P., Berzins K., Calam R., Pryjmachuk S. \& Abel K. M. (2013) Defining Quality of Life in the Children of Parents with Severe Mental IIIness: A Preliminary Stakeholder-Led Model. PLOS ONE, 8, 9, 1-9.

Bettany-Saltikov J. (2012) How to do a systematic literature review in nursing : a step-bystep guide. Maidenhead: Open University Press, New York.

Brunton G., Wiggins M. \& Oakley A. (2011) Becoming a mother: a research synthesis of women's views on the experience of first time motherhood, University of London.

Retrieved from

http://eppi.ioe.ac.uk/cms/LinkClick.aspx?fileticket=hGTI6U9cFJE\%3d\&tabid=3295\&mid=6 $\underline{166}$ on 16 July 2016.

Foster K., O'Brien L. \& Korhonen T. (2012) Developing resilient children and families when parents have mental illness: A family-focused approach. International Journal of Mental Health Nursing 21, 3-11.

Fudge E. \& Mason P (2004) Consulting with young people about service guidelines relating to parental mental illness. Australian e-Journal for the Advancement of Mental Health 2, 1-9.

Fjone H. H., Ytterhus B. \& Almvik A. (2009) How Children with Parents Suffering from Mental Health Distress Search for 'Normality' and Avoid Stigma To be or not to be... is not the question. Childhood 16, 4, 461-477.

Garley D., Gallop R., Johnston N. \& Pipitone J. (1997) Children of the mentally ill: a qualitative focus group approach. Journal of Psychiatric \& Mental Health Nursing 4, 2, 97 103.

Gladstone B. M., Boydell K. M. and McKeever P. (2006) Recasting research into children's experiences of parental mental illness: beyond risk and resilience. Social Science and Medicine 62, 10, 2540-2550.

Gladstone B. M., Boydell K. M., Seeman M. V. \& McKeever P. D. (2011) Children's experiences of parental mental illness: a literature review. Early Intervention in Psychiatry $5,4,271-289$.

Gladstone B. M., McKeever P., Seeman M. and Boydell K. M. (2014) Analysis of a support group for children of parents with mental illnesses: managing stressful situations. Qualitative Health Research 24, 9, 1171-82. 
Grant G., Repper J. \& Nolan M. (2008) Young people supporting parents with mental health problems: experiences of assessment and support. Health \& Social Care in the Community, 16, 3, 271-281.

Hayman F. M. (2009) Kids with confidence: A program for adolescents living in families affected by mental illness. Australian Journal of Rural Health, 17, 5, 268-272.

Hetherington R., Baistow K., Katz I., Mesie J. \& Trowell J. (2001) The welfare of children with mentally ill parents: Learning from inter-country comparisons. John Wiley \& Sons, West Sussex.

Kaimal G. \& Beardslee W. R. (2010). Emerging adulthood and the perception of parental depression. Qualitative Health Research 20, 9, 1213-1228.

Lauritzen C. \& Reedtz C. (2013) Support for children of service users in Norway. Mental Health Practice 16, 10, 12-18.

Leschied A. W., Chiodo D., Whitehead P. C. \& Hurley D. (2005) The relationship between maternal depression and child outcomes in a child welfare sample: implications for treatment and policy. Child \& Family Social Work 10, 4, 281-291.

Maybery D. J., Reupert A. E., Patrick K., Goodyear M. \& Crase L. (2009) Prevalence of parental mental illness in Australian families. Psychiatric Bulletin 33,1, 22-26.

Maybery D. \& Reupert A. (2009) Parental mental illness: a review of barriers and issues for working with families and children, Journal of Psychiatric \& Mental Health Nursing 16, 9, 784-791.

Meadus R. J. \& Johnson B. (2000) The experience of being an adolescent child of a parent who has a mood disorder. Journal of Psychiatric \& Mental Health Nursing 7, 5, 383-390.

Mordoch E. \& Hall W. A. (2008) Children's Perceptions of Living With a Parent With a Mental IIlness: Finding the Rhythm and Maintaining the Frame. Qualitative Health Research 18, 8, 1127-1144.

Noblit G. \& Hare R. (1988) Meta- Ethnography: Synthesising Qualitative Studies. Sage Publications, Newbury Park.

Nicholson J., Biebel K., Hinden B. R., Henry A. D. \& Stier L. (2001) Critical issues for parents with mental illness and their families. Department of Psychiatry, Center for Mental Health Services Research, Univeristy of Massachuseff.

O'Brien L., Anand M., Brady P. \& Gillies D. (2011) Children visiting parents in inpatient psychiatric facilities: Perspectives of parents, carers, and children. International Journal of Mental Health Nursing 20, 2, 137-143.

Orel N. A., Groves P. A. and Shannon L. (2003) Positive Connections: A programme for children who have a parent with a mental illness. Child \& Family Social Work 8, 2, 113122.

Östman M. (2008) Interviews with children of persons with a severe mental illnessInvestigating their everyday situation. Nordic Journal of Psychiatry 62, 5, 354-359.

Pitman P. S E. \& Matthey H. (2004) Coping and resilience of children of a mentally ill parent. Social Work in Health Care 39, 1/2, 151-163. 
Pretis M. \& Dimova A. (2008) Vulnerable children of mentally ill parents: towards evidence-based support for improving resilience. Support for Learning 23, 3, 152-159.

Riebschleger J. (2004) Good days and bad days: the experiences of children of a parent with psychiatric disability. Psychiatric Rehabilitation Journal 28, 1, 25-31.

Rober P. (2002) Some hypotheses about hesitations and their nonverbal expression in family therapy practice. Journal of Family Therapy 24, 2, 187-204.

Seers K. (2012) What is qualitative synthesis? Evidenced Based Nursing 15, 4, 101.

Seers K. (2015) Qualitative systematic reviews: their importance forour understanding of research relevant to pain. British Journal of Pain 9, 1, 36 - 40.

Stallard P., Norman P., Huline-Dickens S., Salter E. \& Cribb J. (2004) The Effects of Parental Mental Illness Upon Children: A Descriptive Study of the Views of Parents and Children. Clinical Child Psychology and Psychiatry 9, 1, 39-52.

Tong A., Sainsbury P. \& Craig J. (2007) Consolidated criteria for reporting qualitative research (COREQ): a 32-item checklist for interviews and focus groups. International Journal for Quality in Healh Care 19, 6, 349-357.

Trondsen M. V. (2012) Living With a Mentally III Parent: Exploring Adolescents' Experiences and Perspectives. Qualitative Health Research 22, 2, 174-188.

Tunnard J. (2004) Parental Mental Health Problems: Key Messages from Research, Policy and Practice. Research in Practice, Devon.

Van Parys, H. and Rober, P. (2013) Trying to comfort the parent: A qualitative study of children dealing with parental depression. Journal of Marital and Family Therapy 39, 3 , pp.330-345. 